\title{
Determining the Fixed Pattern Noise of a CMOS Sensor: Improving the Sensibility of Autonomous Star Trackers
}

\author{
Eduardo dos Santos Pereira ${ }^{1}$
}

\begin{abstract}
Autonomous star trackers are optical-electronic devices used for attitude determination of artificial satellites, having as a reference for this computation the positions of stars. There is one autonomous star tracker in development at the Aerospace Electronics Division of the Brazilian National Institute for Space Research. The autonomous star tracker imager is a complementary metal-oxide-semiconductor active pixel sensor, consisting of an integrated circuit with an array of them. Each pixel has a photodetector and an active amplifier. Since it has many amplifiers, the active pixel sensor has an additional fixed pattern noise, therefore its characterization is different from the traditional method used for the charge coupled devices. With this experiment, it was observed that the mean value per columns of fixed pattern noise is 100\% greater than the mean value of the pixel one. Taking into account this result, modeling the pixel fixed pattern noise will not be important to improve the autonomous star tracker sensibility. Furthermore, the random noise value was less than $1 \%$ of the fixed pattern noise range data, being possible to estimate it. In this work, we presented the fixed pattern noise new parametric model, which has a good agreement when compared with the experimental data. It was calculated Pearson's product-moment correlation coefficient between the model and the observed data, in order to quantify the model accuracy and it was obtained $99 \%$ for flat field and $79 \%$ for dark current.
\end{abstract}

KEYWORDS: Star trackers, Aerospace systems, Attitude determination, Spacecraft, Applied astronomy.

\section{INTRODUCTION}

Autonomous star trackers (AST) are optoelectronic instruments used for attitude determination of a satellite by observing stars (Liebe, 1995, Liu et al., 2011). The precision of attitude determination depends on the accuracy of the AST image registration. Thus, the calibration of a fixed pattern noise (FPN) of the AST image sensor has an important role. The FPN is a variation in the output pixel values, under uniform illumination, due to device and interconnection mismatches across an image sensor. In the case of complementary metaloxide-semiconductor (CMOS) sensors, passive and active pixel (PPS and APS), there are several amplifiers in which some are shared by pixels and others are not. Thereby, in order to determine the FPN, it is necessary to take into account not only the pixel noise, but also that of the column (Gamal et al., 1998, Bigas et al., 2006). On the other hand, Schöberl et al. (2009) showed that it is possible to model the FPN as a function of the image acquisition integration time, with a nonlinear parametric model. However, for that model, it is necessary to find a set of parameters for each pixel, and for a 6 MPixel sensor 75 Mbytes are required to save those data. Another issue presented by Schöberl et al. (2009) is concerning the fact of assuming dark current as a constant for each pixel as the model of Pillman et al. (2006) for describing the FPN with a linear algorithm. However, these authors also presumed that the segmented linear and quadratic models have a better characterization of the FPN than a pure linear one.

In this work, we were mainly interested in determining and modeling the FPN of the CMOS used in the AST. This 
procedure would allow us to perform a data correction before starting the process of pattern recognition from stars. Such review will improve the sensibility of the AST and therefore reduce errors on the satellite attitude determination.

\section{AUTONOMOUS STAR SENSOR}

The AST consists of a pinhole imaging system that measures the direction vector of a star in its own reference frame (Zenick, 2003, Xing et al., 2006, Liu et al., 2011). Firstly, the stars into the field of view (FOV) are registered by the CMOS APS, secondly they are recognized by a pattern recognition routine. Finally, the stars are compared with an internal catalog and the attitude matrix of the AST is calculated. In Fig. 1 a simplified block diagram of AST functionality is presented.

For the calculus of AST attitude matrix, $M$, it is necessary to know a vector direction, $v$, of the star in an inertial frame. The value of $v$ is obtained from the right ascension, $\alpha$, and declination, $\delta$, from a master catalog, as Eq. 1 :

$v=\left[\begin{array}{c}v_{x} \\ v_{y} \\ v_{z}\end{array}\right]=\left[\begin{array}{c}\cos (\alpha) \cos (\delta) \\ \sin (\alpha) \cos (\delta) \\ \sin (\delta)\end{array}\right]$.

Then, a new catalog is generated. The attitude matrix should satisfy (Eq. 2):

$W=M v \quad$,

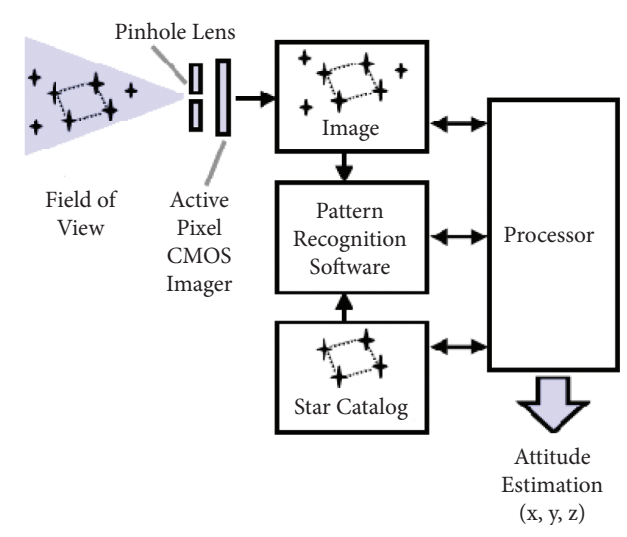

Figure 1. Simplified block diagram of a star tracker. Adapted from Zenick (2003). where $W$ is the vector direction of stars into the AST reference frame, which is given by Eq. 3 (Xing et al., 2006 and Liu et al., 2011):

$W=\frac{1}{\sqrt{\left(x-x_{0}\right)^{2}\left(y-y_{0}\right)^{2}+f^{2}}}\left[\begin{array}{c}-(x-x 0) \\ -(y-y 0) \\ f\end{array}\right]$,

where $x_{0}$ and $y_{0}$ represent the intersection points of the focal plane and the optical axis; $x$ and $y$ are the observed star locations on the detector plane and $f$ is the focal length of the AST camera. In Fig. 2 an illustration of the AST reference, $o$, and inertial frames, $O^{\prime}$, is presented.

Usually, the matrix $M$ can be estimated by minimizing the least-square error (Eq. 4):

$L=\frac{1}{2} \sum_{i=1}^{n} a_{i}^{\prime}\left\|M v_{i}-W_{i}\right\|^{2}$,

where $a_{\mathrm{i}}^{\prime}$ are the weights and $\mathrm{n}$ is the number of observations. This equation is also called Wahba's problem (Wahba, 1965).

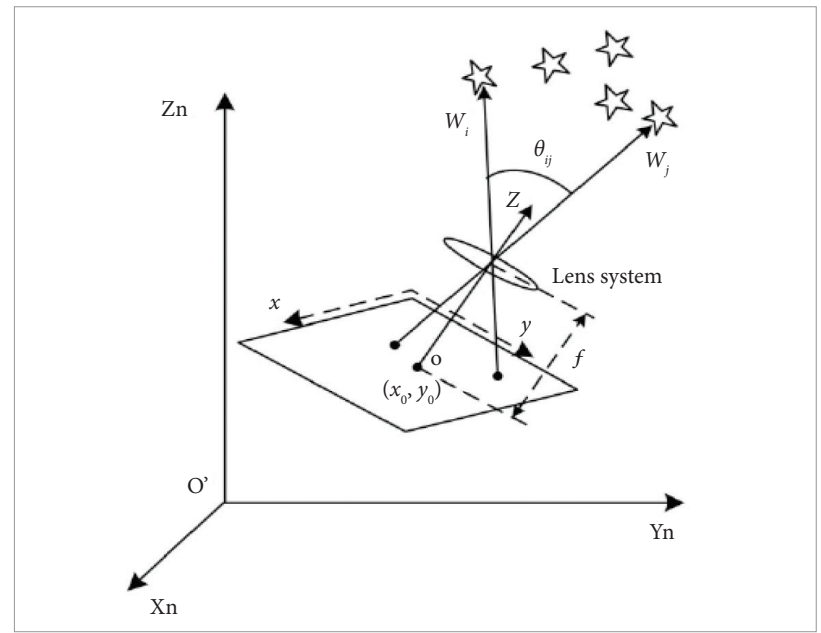

Figure 2. Star tracker measurement. The 0 represents the inertial referential frame and $O$ ' is the autonomous star trackers referential frame. Adapted from Liu et al. (2011).

\section{ESTIMATION AND MODELING THE FIXED PATTERN NOISE}

To estimate the FPN, we started considering the method described by Gamal et al. (1998). Thus, from the sample of images, the FPN is determined by column and pixel, which are in Eqs. 5 and 6, respectively: 


$$
\begin{aligned}
& \bar{Y}_{j}=\frac{1}{N} \sum_{i=1}^{N} F_{i j}, \\
& \overline{X_{i j}}=F_{i j}-\bar{Y}_{j},
\end{aligned}
$$

where $F_{\mathrm{ij}}$ is an average estimator that is obtained from a sample of AST images, and $N$ is the number of columns of the images. $F_{\mathrm{ij}}$ is determined as follows:

- obtain a sample of $\mathrm{k}$ images from the AST;

- in order to reduce the random noise, the mean value of the sample should be calculated;

- from the result of the image, the average value of all pixels is found;

- $F_{\mathrm{ij}}$ is determined through the subtraction from the mean image of the average value of all their pixels, each at a time.

The variances, by column and pixel, are presented in Eqs. 7 and 8 :

$$
\begin{aligned}
& \bar{\sigma}_{Y}^{2}=\frac{1}{M-1} \sum_{j=1}^{M} \bar{Y}_{j}^{2}, \\
& \bar{\sigma}_{x}^{2}=\frac{1}{M(N-1)} \sum_{i=1}^{N} \sum_{j=1}^{M} \bar{X}_{i j}^{2} .
\end{aligned}
$$

Furthermore, we consider the normalized root mean square error (NRMSE) for each image of the sample in order to determine the predominance of the random error. Therefore, this allows us to know if it is possible to make a FPN correction. In the present case, a residual value of each image, $R_{i j}^{k}$, was obtained by subtracting the average value of all the pixels, each at a time, from the image $k$. The NRMSE of FPN by columns and pixels is in Eqs. 9 and 10:

$$
\begin{aligned}
& C_{r m s}^{k}=\frac{1}{\max (\bar{Y})-\min (\bar{Y})} \sqrt{\frac{1}{N} \sum_{j=1}^{N}\left(\frac{1}{N} \sum_{i=1}^{N} R_{i j}^{k}-\overline{Y_{j}}\right)^{2}} \\
& P_{r m s}^{k}=\frac{1}{\max \left(\overline{X_{j}}\right)-\min \left(\overline{X_{j}}\right)} \sqrt{\frac{1}{M N} \sum_{i=1}^{M} \sum_{j=1}^{N}\left(R_{i j}^{k}-\frac{1}{N} \sum_{i=1}^{N} R_{i j}^{k}-\overline{X_{i j}}\right)^{2}}
\end{aligned}
$$

In this work, we modeled the FPN columns tendency as a third-order polynomial function (Eq. 11),

$$
f_{c}(x)=a_{0}+a_{1} x+a_{2} x^{2}+a_{3} x^{3},
$$

and based on the work of Schöberl et al. (2009), we considered a fit of the FPN columns as a function also of the integration time as Eq. 12:

$F_{f p n}(x, \tau)=f_{c}(x) g_{c}(\tau)$

where $\tau$ represents the integration time, and $g_{c}(\tau)=b_{0}+b_{1} \tau+b_{2} \tau^{2}$ and $a_{i}, b_{i}$ are parameters to be determined. However, in the work of Schöberl et al. (2009), the set of parameters found were for each individual pixel. Herein, we are more interested in modeling the column FPN using only one function for all columns and integration time range.

In order to determine $a_{\mathrm{i}}, b_{\mathrm{i}}$, we minimize the least-square error as in Eq. 13:

$J(\mathrm{a}, \mathrm{b})=\frac{1}{2} \sum_{l} \sum_{j}\left\|\overline{Y_{j l}}-F_{f p n}\left(\mathrm{a}, \mathrm{b}, x_{j}, \tau_{l}\right)\right\|^{2}$,

where $\mathrm{a}=\left[a_{0}, a_{1}, a_{2}, a_{3}\right], \mathrm{b}=\left[b_{0}, b_{1}, b_{2}\right]$, and $\overline{Y_{j l}}$ are the FPN columns given by Eq. 13 for $l$ different integration times.

In order to quantify the model fit accuracy, using Eq. 12 and setting the best fit parameters to compare with the experimental data, we have considered Pearson's product-moment correlation coefficient (Press et al., 1993), as seen in Eq. 14:

$\rho=\frac{\sum_{j} \sum_{l}\left(\overline{Y_{j l}}-\bar{Y}\right)\left(F_{f p n, j l}-F_{f p n}\right)}{\left.\sqrt{\sum_{j} \sum_{l}\left(\bar{Y}_{j l}-\bar{Y}\right)^{2}} \sqrt{\sum_{j} \sum_{l}\left(F_{f p n, j l}-\overline{F_{f p n}}\right.}\right)^{2}}$,

being $\bar{Y}$ the mean value of $\overline{Y_{j l}}, F_{\mathrm{fpn}, \mathrm{jl}}$ is given by Eq. 12 for $x_{j}, \tau_{1}$ and $\overline{F_{f p n}}$ is the mean value of $F_{\mathrm{fpn}, \mathrm{jl}}$.

\section{RESULTS}

We collect the images sample under dark and flat field illuminations. In both cases, we consider 100, 200, 300 and $400 \mathrm{msec}$. of integration time. For the flat field it was used an integrating sphere, which is a device for measuring optical radiation. For more details see the technical manual (http:// www.photonicsonline.com/doc.mvc/A-Guide-to-IntegratingSphere-Radiometry-and-0001). The mean value of the FPN per column is $\sim 100$ greater than that of the pixel FPN. Thus, the modeling of pixel FPN will not be important to improve the 
AST sensibility. Taking into account these results, in this work we focused our attention on the column FPN modeling.

Figure 3 shows the total FPN estimated, $F_{\mathrm{ij}}$, in (A) and the pixel one, in (B). It is noteworthy that the value of the pixel FPN is the $F_{\mathrm{ij}}$ subtracted from the column FPN. These results are for 200 msec of integration time. It is also possible to observe vertical lines in the image in A, which are the FPN column, and their values are plotted in Fig. 4. The highlighted line in Fig. 3 is a defective column in our APS. This column is not considered for the FPN analyses.

Figures 4 and 5 show the FPN per columns for dark current (flat field), the continuous line is the polynomial modeling of the column FPN, given by Eq. 11. In these cases, the vector of parameters that provide us a better fit is presented in Table 1 .

In Figs. 6 and 7 the normalized root mean square error of FPN per column estimated for each image of the sample for dark current and flat field is represented. In both cases, the integration time was $200 \mathrm{msec}$. These figures represent the general behavior of the random noise from image to image, when compared to the FPN range amplitude. In general, the random noise, or error of FPN, has a value lower than $1 \%$ with respect to FPN range amplitude.

The Figs. 8 and 9 demonstrate the last-square fit of the Eq. 12 for dark current and flat field, respectively. The set of best fit parameters are given in Table 2.

Pearson's product-moment correlation coefficients, given by Eq. 14, were obtained for dark current (0.7947) and flat field (0.9995).

These results show that we had a good agreement with the modeling fit when compared with the experimental data. This means that we have $99.95 \%$ of accuracy in modeling the flat field column FPN and 79,47\% of accuracy in the dark current model for the column FPN.

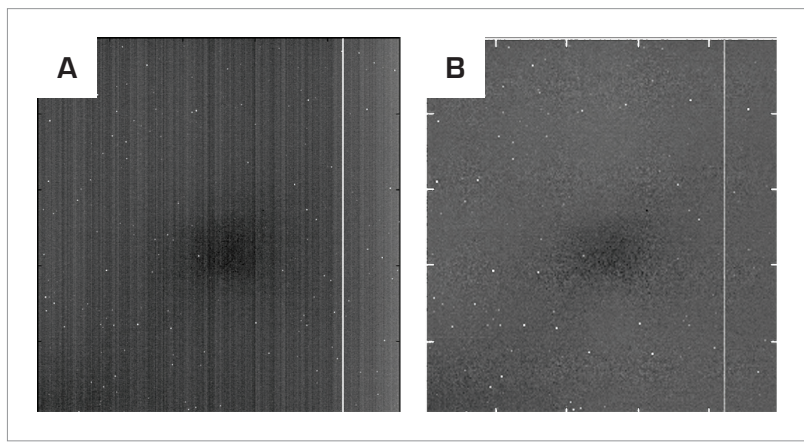

The bright column to the right is the defective of the APS, which is not being taking into account for the fixed pattern noise modeling.

Figure 3. High brightness and contrast image of dark current for 200 msec of integration time. Total fixed pattern noise estimation $(A)$ and pixel fixed pattern noise $(B)$.
For this work, a software tool, called FPNAnalyser ${ }^{\complement}$ was developed for analyzing and modeling the FPN. This program was based on all the theories about FPN determination presented here. The first tab of the Graphical Using Interface of FPNAnalyser ${ }^{\complement}$ is shown in Fig. 10. It will be released as an

Table 1. Best fit parameters set for modeling fixed pattern noise columns.

\begin{tabular}{|l|c|c|c|c|} 
& $\mathrm{a}_{0}$ & $\mathrm{a}_{1}$ & $\mathrm{a}_{2}$ & $\mathrm{a}_{3}$ \\
\hline Dark current & 81.9 & $8.5 \times 10^{-3}$ & $-1.1 \times 10^{-4}$ & $2.0 \times 10^{-7}$ \\
\hline Flat field & 168.0 & $5.5 \times 10^{-2}$ & $-3.5 \times 10^{-4}$ & $4.3 \times 10^{-7}$ \\
\hline
\end{tabular}

The column fixed pattern noise (FPN) has different behaviors when the dark current FPN is compared to the flat field one. As the FPN is distinct for each sensor, we expect different sets of parameters for varied complementary metaloxide-semiconductor sensors.

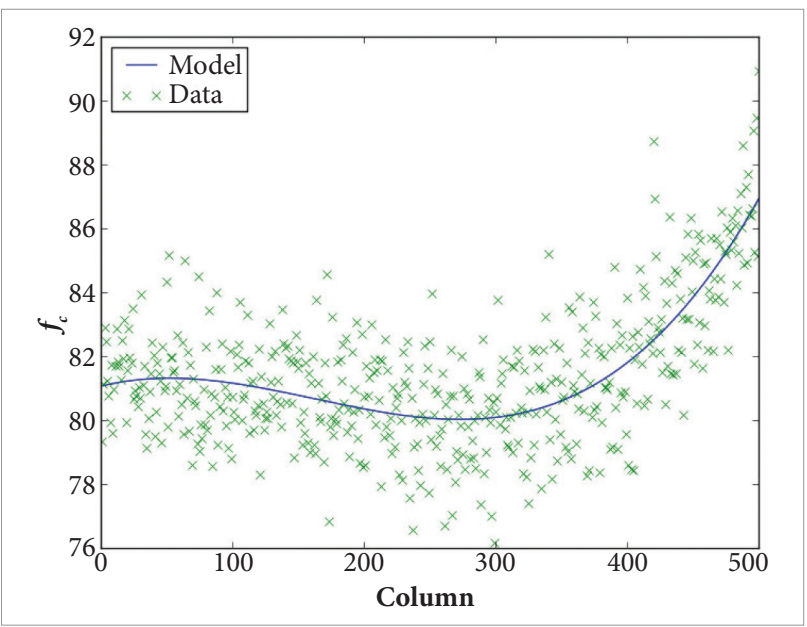

The continuous line is the polynomial modeling.

Figure 4. Column fixed pattern noise for dark current and 200 msec of integration time.

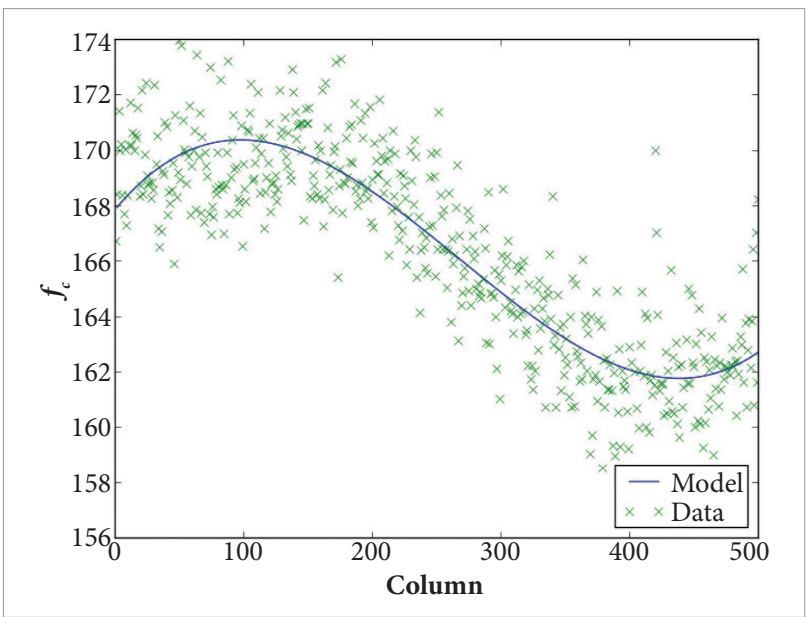

The continuous line is the polynomial modeling.

Figure 5. Column fixed pattern noise for flat field and 200 msec of integration time. 


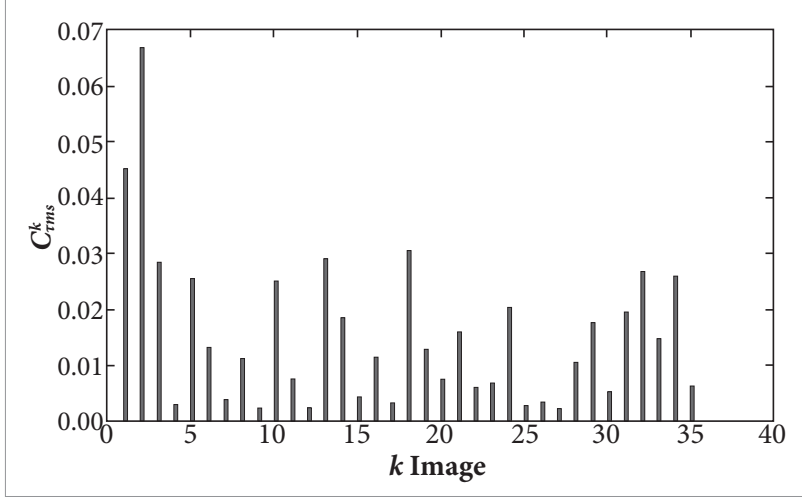

Figure 6. Normalized root mean square error for dark current and 200 msec of integration time.

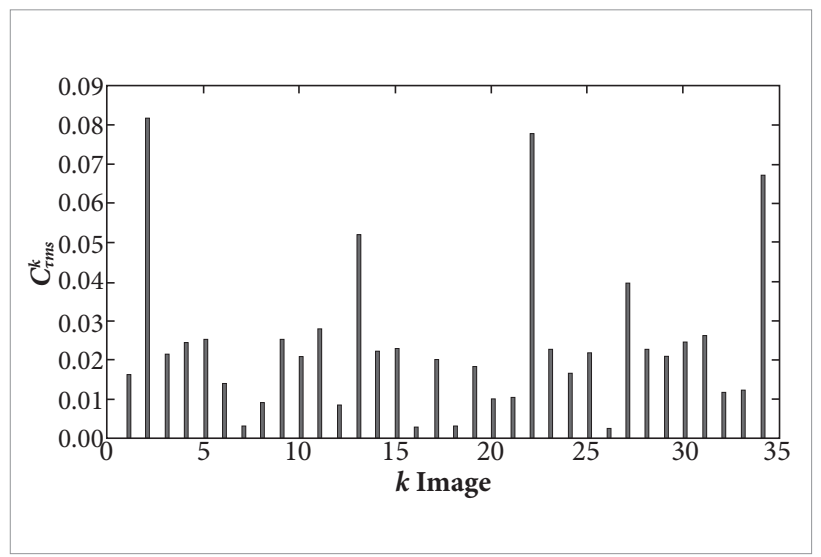

Figure 7. Normalized root mean square error for flat field and 200 msec of integration time.

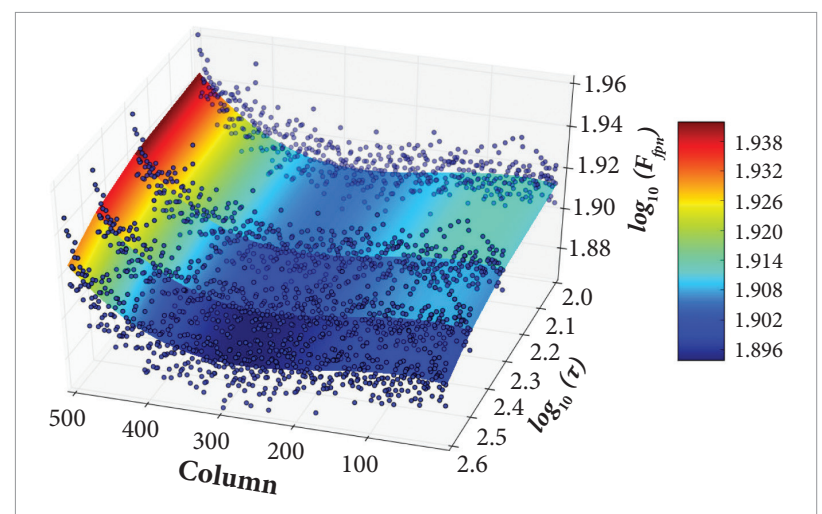

The integrating time, $\tau$, is in msec. This surface was obtained using Eq. 13 The scattered points are experimental data.

Figure 8. Least-square fit of the fixed pattern noise column considering dark current.

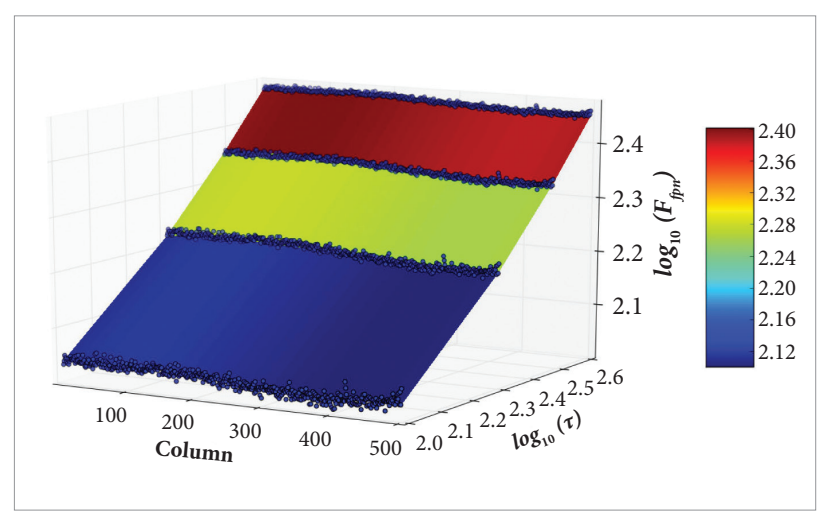

The integrating time, $\tau$, is in msec. This surface was obtained using Eq. 13. The scattered points are experimental data.

Figure 9. Least-square fit of the fixed pattern noise column considering flat field.

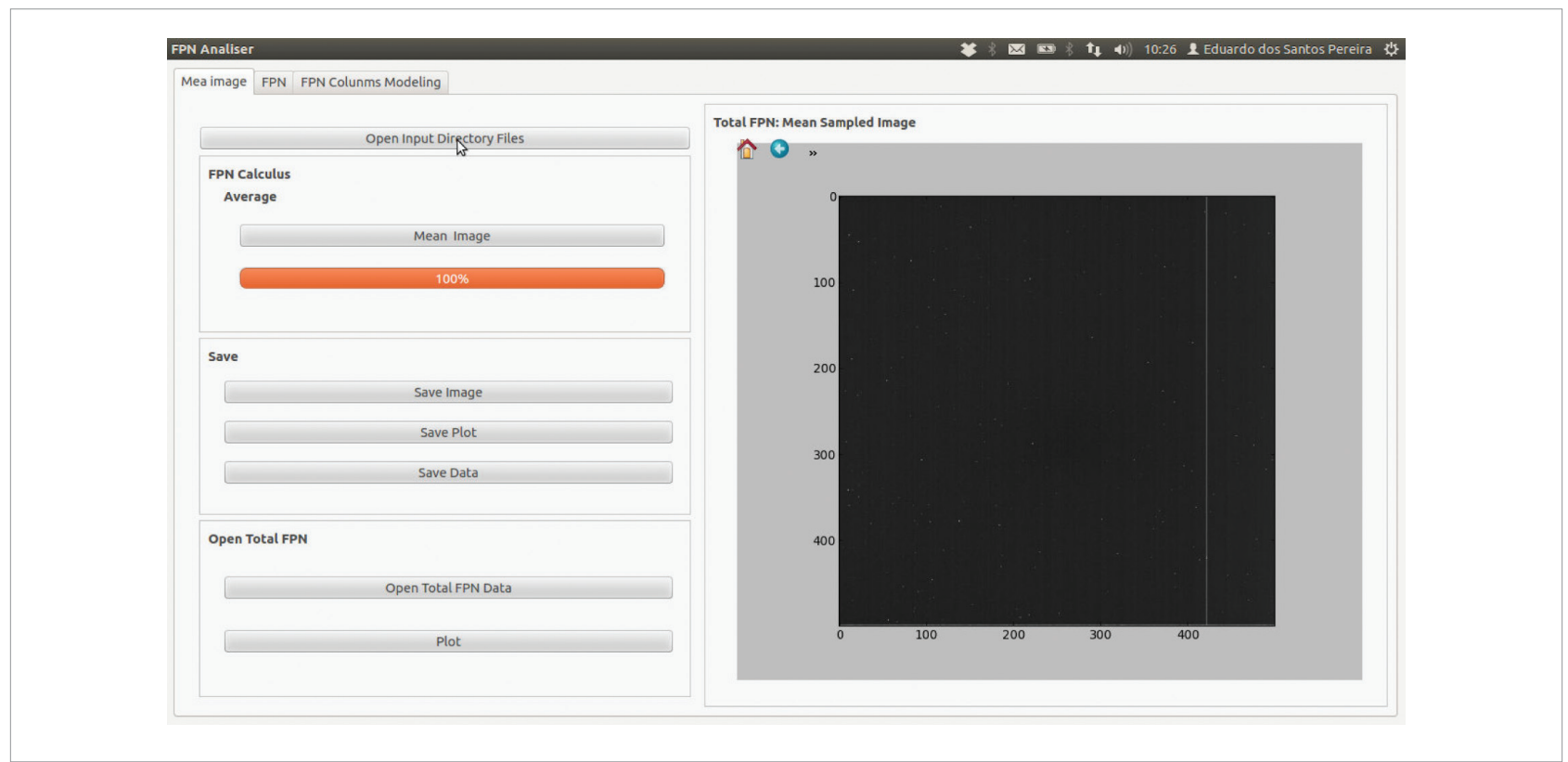

Figure 10. Graphic using interface of the FPNAnalyser ${ }^{\circledR}$ software. 
Table 2. Set of best fit parameters for fixed pattern noise columns models as a functional of integration time.

\begin{tabular}{|l|c|c|c|c|c|c|c|}
\hline & $a_{0}$ & $a_{1}$ & $a_{2}$ & $a_{3}$ & $b_{0}$ & $b_{1}$ & $b_{2}$ \\
\hline Dark current & 7.3 & $1.0 \times 10^{-3}$ & $-1.1 \times 10^{-5}$ & $2.3 \times 10^{-8}$ & 11.2 & $9.7 \times 10^{-5}$ & $-3.3 \times 10^{-6}$ \\
\hline Flat field & -5.2 & $-1.3 \times 10^{-3}$ & $8.3 \times 10^{-5}$ & $-1.0 \times 10^{-8}$ & -10.7 & $-1.0 \times 10^{-1}$ & $-2.9 \times 10^{-6}$ \\
\hline
\end{tabular}

open source under the general GNU license version 3 (see http://www.gnu.org/licenses/gpl-3.0.txt for more details).

\section{DISCUSSION AND CONCLUSION}

It was presented a general concept of AST and a way to evaluate the FPN of CMOS APS used as the AST image. The determination of the FPN is important to achieve not only higher precision of observations of greater magnitude stars (less bright stars), but also to perform corrections of the brightness of low magnitude stars. The attitude determination depends on the precision of stellar identification, therefore the FPN correction leads to a better knowledge of this attitude.

With this experiment, it was observed that the mean value per FPN columns is greater than that of the pixel FPN. Thus, it is relevant only the FPN column for doing the APS image correction. Also, we showed that the parametric model of FPN columns, as function of columns and integration time, had a good agreement with experimental data. This fact was quantified by Pearson's product-moment correlation coefficient. We obtained $99.95 \%$ of accuracy for flat field and a $79.47 \%$ for dark current. We strongly suggest future works including the development and implementation of the correction algorithm of FPN columns for the AST.

The greatest contribution of this work is the applied methodology, since it was widely detailed in a single paper, i.e., a FPN correction analysis for an AST image. Another point is that we have developed a software tool that could be used not only for modeling the FPN, but also to trace strategies for doing an automatic image correction by an embedded system into the AST image.

\section{ACKNOWLEDGEMENTS}

Eduardo S. Pereira would like to thank the Conselho Nacional de Desenvolvimento Científico e Tecnológico (CNPQ) for their financial support (process: 382477/2012), Instituto Nacional de Pesquisas Espaciais (INPE) for their technical support, and Regla Duthit Somoza and Marcio A. A. Fialho for their constructive opinions.

\section{REFERENCES}

Bigas, M., Cabruja, E., Forest, J., Salvi, J., 2006, "Review of CMOS image sensors”, Microelectronics Journal, Vol. 37, pp. 433-451.

Gamal, A.E., Fowler, B., Min, H., Liu, X., 1998, "Modeling and estimation of FPN components in CMOS image sensors", International Society for Optics and Photonics, Vol. 3301, pp. 168-177.

Liebe, C.C., 1995, "Star trackers for attitude determination", Aerospace and Electronic Systems Magazine, Institute of Electrical and Electronics Engineers, Vol. 10, pp. 10-16.

Liu, H.B., Wang, J., Tan, J., Yang, J., Jia, H., Li, X., 2011, "Autonomous on-orbit calibration of a star tracker camera", Optical Engineering, Vol. 50, pp. 023604.

Pillman, B., Guidash, R., Kelly, S., 2006, "Fixed pattern noise removal in CMOS imagers across various operational conditions", US Patent 7,092,017.
Press, W.H., Teukolsky, S.A., Vetterling, W.T., Flannery, B.P., 1993, "Numerical Recipes in FORTRAN; The Art of Scientific Computing", Cambridge University Press, New York, NY, USA, 973 p.

Schöberl, M., Senel, C., Fößel, S., Bloss, H., Kaup, A., 2009. "Nonlinear Dark Current Fixed Pattern Noise Compensation for Variable Frame Rate Moving Picture Cameras", 17th European Signal Processing Conference (EUSIPCO), Vol. 1, Glasgow, Scotland, pp. 268-272.

Wahba, G., 1965, "A least squares estimate of satellite attitude", SIAM Review, Vol. 7, pp. 409-409.

Xing, F., Dong, Y., You, Z., 2006, "Laboratory calibration of star tracker with brightness independent star identification strategy", Optical Engineering, Vol. 45, pp. 063604-063604-9.

Zenick, R., 2003, "Lightweight, low-power coarse star tracker", Proceedings of the AIAA/USU Conference on Small Satellites, Mission Lessons, SSCO3-X-7, Vol. 1, Logan, Utah, pp. 01-14. 